\title{
Coulomb breakup of two-neutron halo nuclei
}

\author{
P. Banerjee, J. A. Tostevin, and I. J. Thompson \\ Department of Physics, School of Physical Sciences, University of Surrey, Guildford, Surrey GU2 5XH, United Kingdom
}

(Received 30 March 1998)

\begin{abstract}
We investigate the Coulomb dissociation of two-neutron halo nuclei, assuming that the excitation of the projectile is to states in the continuum with low energy. The method presented retains all finite-range effects associated with the interactions between the breakup fragments and can use realistic three-body wave functions for the two-neutron halo nuclei. The method is applied to ${ }^{6} \mathrm{He}$, at incident energies below $100 \mathrm{MeV} /$ nucleon, and calculations are compared with available data for the $\alpha$-particle energy spectrum following the breakup of ${ }^{6} \mathrm{He}$ on a heavy target. [S0556-2813(98)01308-9]
\end{abstract}

PACS number(s): 24.10.Eq, 25.60.-t, 25.70.Mn

Two-neutron halo nuclei, characterized by small twoneutron separation energies, have recently been the subject of considerable theoretical and experimental attention [1-3]. Particular interest has centered on the possible existence of a soft electric dipole excitation, associated with low-energy oscillation of the two loosely bound neutrons with respect to the core nucleons [4]. For ${ }^{11} \mathrm{Li}$ there have been several experimental studies using Coulomb excitation on a heavy target [5]. For ${ }^{6} \mathrm{He}$ such a mode has been discussed theoretically [6-8] but remains to be observed empirically.

There have been several theoretical analyses, both semiclassical [9] and quantum mechanical $[10,11]$, of the Coulomb breakup of two-neutron halo nuclei-particularly for ${ }^{11} \mathrm{Li}$. These calculations were not, however, based upon realistic three-body wave functions. Instead, the two halo neutrons were treated as a "dineutron" cluster orbiting the core. In addition, a zero-range approximation was assumed in the dineutron-core interaction.

In this paper we present new quantum-mechanical calculations of the Coulomb breakup of the two-neutron halo nucleus ${ }^{6} \mathrm{He}$. Our analysis is a extension of the work of Ref. [12] for three-body projectiles where only a single constituent, the core, is charged. The method presented will be seen to treat fully the finite-range of the interactions between the projectile constituents, before and after dissociation. It also permits the use of realistic correlated three-body wave functions for the halo nuclei.

We will consider the Coulomb dissociation of a twoneutron halo nucleus $a$ by a heavy charged target $t$ within a four-body model. The incident projectile's ground state will be described by a correlated three-body wave function $\Phi_{a}(\boldsymbol{\rho}, \boldsymbol{r})$ for the relative motions of the two halo neutrons, 1 and 2 , and the assumed structureless charged core $c$. For ${ }^{6} \mathrm{He}$, and also for ${ }^{11} \mathrm{Li}$, the total angular momentum $J$ of the two halo neutrons is zero. Additionally we will assume that only the core interacts with the target through a spinindependent point Coulomb interaction $V_{c t}\left(R_{c}\right)$. We adopt the coordinates shown in Fig. 1.

Within this model, the elastic breakup amplitude in the center of mass (c.m.) frame, from an incident projectile state $\boldsymbol{k}_{a}$ to a four-body final state with a core momentum $\hbar \boldsymbol{k}_{c}$, and the two-neutrons having a c.m. wave number $\boldsymbol{k}_{v}$, relative motion wave number $\boldsymbol{k}$, total spin $S$, and projection $\sigma$ is

$$
T_{S \sigma}=\left\langle\chi_{\boldsymbol{k}_{c}}^{(-)}\left(\boldsymbol{R}_{c}\right) e^{i \boldsymbol{k}_{v} \cdot \boldsymbol{R}_{v}} e^{i \boldsymbol{k} \cdot \boldsymbol{r}} \mathcal{X}_{S \sigma}|\mathcal{V}(\boldsymbol{\rho}, \boldsymbol{r})| \Psi_{\boldsymbol{k}_{a}}^{(+)}(\boldsymbol{\rho}, \boldsymbol{r}, \boldsymbol{R})\right\rangle .
$$

Here $\Psi_{\boldsymbol{k}_{a}}^{(+)}$is the exact solution of the Schrödinger equation for the four-body model Hamiltonian, $\chi_{\boldsymbol{k}_{c}}^{(-)}$is a Coulomb distorted wave for the core in the final state, and $\mathcal{X}_{S \sigma}$ is the total spin wave function for the two neutrons. $\mathcal{V}(\boldsymbol{\rho}, \boldsymbol{r})$ is the transition interaction

$$
\mathcal{V}(\boldsymbol{\rho}, \boldsymbol{r})=V_{12}(\boldsymbol{r})+V_{c 1}(\boldsymbol{\rho}-\boldsymbol{r} / 2)+V_{c 2}(\boldsymbol{\rho}+\boldsymbol{r} / 2),
$$

the sum of the two-body interactions between the projectile constituents.

The differential cross section for elastic breakup is therefore

$$
\begin{aligned}
& \frac{d^{5} \sigma}{d E_{c} d \Omega_{c} d E_{1} d \Omega_{1} d \Omega_{2}} \\
& =\frac{2 \pi}{\hbar v_{a}}\left[\sum_{S \sigma}\left|T_{S \sigma}\right|^{2}\right] \rho\left(E_{c}, \Omega_{c}, E_{1}, \Omega_{1}, \Omega_{2}\right),
\end{aligned}
$$

where $\rho(\cdots)$ is the four-body phase space for the frame (laboratory or c.m.) of interest and $v_{a}$ is the $a-t$ relative velocity in the entrance channel.

If now one assumes that the excitation of the projectile $a$ can be treated adiabatically, i.e., that the breakup configurations excited by $V_{c t}$ are low-energy $c+1+2$ relative motion

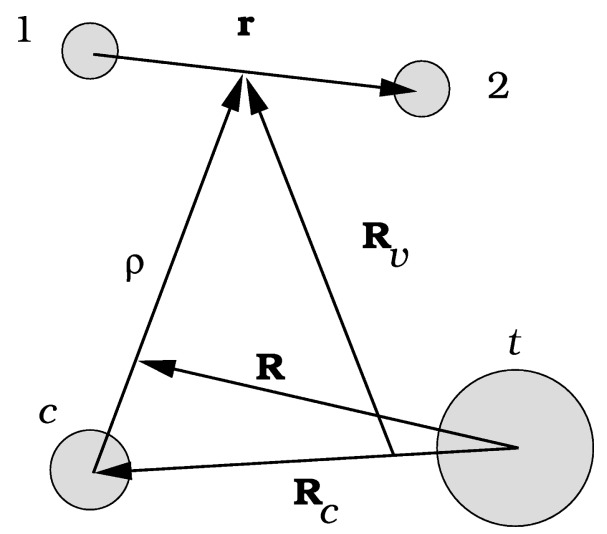

FIG. 1. Definition of the coordinate vectors used. 
states, then, following the analysis of Refs. [12,13], the adiabatic (AD) approximation to the four-body wave function $\Psi_{\boldsymbol{k}_{a}}^{(+)}$has a particularly simple closed form,

$$
\Psi_{\boldsymbol{k}_{a}}^{\mathrm{AD}}(\boldsymbol{\rho}, \boldsymbol{r}, \boldsymbol{R})=\Phi_{a}(\boldsymbol{\rho}, \boldsymbol{r}) e^{i \gamma \boldsymbol{k}_{a} \cdot \boldsymbol{\rho}} \chi_{\boldsymbol{k}_{a}}^{(+)}\left(\boldsymbol{R}_{c}\right)
$$

Here $\gamma=2 m_{n} /\left(2 m_{n}+m_{c}\right)$ is the ratio of the masses of the valence particles and the projectile, and $\chi_{\boldsymbol{k}_{a}}^{(+)}$is a Coulomb distorted wave for the incident projectile, but evaluated at coordinate $\boldsymbol{R}_{c}$ and not at its center of mass $\boldsymbol{R}$. Our adiabatic approximation to the elastic breakup amplitude is obtained upon replacing $\Psi_{\boldsymbol{k}_{a}}^{(+)}$by $\Psi_{\boldsymbol{k}_{a}}^{\mathrm{AD}}$ in Eq. (1).

It follows, since $\boldsymbol{R}_{v}=\alpha \boldsymbol{R}_{c}+\boldsymbol{\rho}$, where $\alpha=m_{t} /\left(m_{c}+m_{t}\right)$, that the adiabatic approximation to the breakup amplitude now factorizes exactly as

$$
\begin{aligned}
T_{S \sigma}^{\mathrm{AD}}= & \left\langle e^{i \mathcal{K} \cdot \boldsymbol{\rho}} e^{i \boldsymbol{k} \cdot \boldsymbol{r}} \mathcal{X}_{S \sigma}|\mathcal{V}(\boldsymbol{\rho}, \boldsymbol{r})| \Phi_{a}(\boldsymbol{\rho}, \boldsymbol{r})\right\rangle \\
& \times\left\langle\chi_{\boldsymbol{k}_{c}}^{(-)}\left(\boldsymbol{R}_{c}\right) e^{i \alpha \boldsymbol{k}_{v} \cdot \boldsymbol{R}_{c}}\right| \chi_{\boldsymbol{k}_{a}^{(+)}}^{\left.\left(\boldsymbol{R}_{c}\right)\right\rangle} \\
\equiv & \left\langle\mathcal{K}, \boldsymbol{k}, S \sigma|\mathcal{V}| \Phi_{a}\right\rangle \times\left\langle\chi_{\boldsymbol{k}_{c}}^{(-)}, \alpha \boldsymbol{k}_{v} \mid \chi_{\boldsymbol{k}_{a}}^{(+)}\right\rangle,
\end{aligned}
$$

where we have introduced the vector $\mathcal{K}=\boldsymbol{k}_{v}-\boldsymbol{\gamma} \boldsymbol{k}_{a}$, the momentum transferred in the breakup to the motion of the c.m. of the two neutrons. We mention that a similar factored expression was obtained in Ref. [14] for a two-body projectile using an effective local momentum approximation in the distorted-wave Born approximation (DWBA) to the breakup amplitude. That expression suffers from an ambiguity in the choice of the direction of this effective momentum.

As is discussed elsewhere [15], the second term in Eq. (5) can be expressed in terms of the bremsstrahlung integral [16] and reflects entirely the dynamical component of the amplitude. The projectile structure enters through a three-body vertex function, the first term in Eq. (5). This is also readily extracted from three-body model calculations of the projectile structure, as follows.

The angular momentum structure of the three-body vertex in configuration space is, e.g., [17],

$$
\begin{aligned}
\left\langle\boldsymbol{\rho}, \boldsymbol{r}|\mathcal{V}| \Phi_{a}\right\rangle= & \sum_{\lambda l L S} \widetilde{\phi}_{\lambda l L S}(\rho, r) \\
& \times\left\{\left[Y_{\lambda}(\hat{\boldsymbol{\rho}}) \otimes Y_{l}(\hat{\boldsymbol{r}})\right]_{L} \otimes \mathcal{X}_{S}\right\}_{J M=00},
\end{aligned}
$$

and hence the vertex function appearing in Eq. (5) is

$$
\begin{aligned}
\left\langle\mathcal{K}, \boldsymbol{k}|\mathcal{V}| \Phi_{a}\right\rangle= & 4 \pi \sum_{\lambda l L S}(-i)^{l+\lambda} \widetilde{\boldsymbol{\phi}}_{\lambda l L S}(\mathcal{K}, k) \\
& \times\left[\left[Y_{\lambda}(\hat{\mathcal{K}}) \otimes Y_{l}(\hat{\boldsymbol{k}})\right]_{L} \otimes \mathcal{X}_{S}\right]_{00},
\end{aligned}
$$

with

$$
\begin{aligned}
& \widetilde{\phi}_{\lambda l L S}(\mathcal{K}, k) \\
& \quad=4 \pi \int_{0}^{\infty} d \rho \rho^{2} \int_{0}^{\infty} d r r^{2} j_{\lambda}(\mathcal{K} \rho) j_{l}(k r) \widetilde{\phi}_{\lambda l L S}(\rho, r) .
\end{aligned}
$$

It follows from Eq. (5) that, when substituted in Eq. (3),

$$
\begin{aligned}
\sum_{S \sigma}\left|T_{S \sigma}^{\mathrm{AD}}\right|^{2}= & {\left[\sum_{S \sigma}\left|\left\langle\boldsymbol{\mathcal { K }}, \boldsymbol{k}, S \sigma|\mathcal{V}| \Phi_{a}\right\rangle\right|^{2}\right] } \\
& \times\left|\left\langle\chi_{\boldsymbol{k}_{c}}^{(-)}, \alpha \boldsymbol{k}_{v} \mid \chi_{\boldsymbol{k}_{a}}^{(+)}\right\rangle\right|^{2} \\
\equiv & \mathcal{D}^{2}(\mathcal{K}, \boldsymbol{k}) \times\left|\left\langle\chi_{\boldsymbol{k}_{c}}^{(-)}, \alpha \boldsymbol{k}_{v} \mid \chi_{\boldsymbol{k}_{a}}^{(+)}\right\rangle\right|^{2} .
\end{aligned}
$$

A general expression for $\mathcal{D}^{2}(\mathcal{K}, \boldsymbol{k})$ can be obtained from Eqs. (6) and (7), and using Eq. (25) of Ref. [17]. For ${ }^{6} \mathrm{He}$, where the dominant angular momentum configurations in the $0^{+}$ground state wave function are the $\phi_{0} \equiv \phi_{0000}(\rho, r)$ $(\approx 84 \%)$ and $\phi_{1} \equiv \phi_{1111}(\rho, r)(\approx 11 \%)$ components, with the indicated probabilities, then

$$
\mathcal{D}^{2}(\mathcal{K}, \boldsymbol{k})=\left[\widetilde{\boldsymbol{\phi}}_{0}^{2}(\mathcal{K}, k)+\widetilde{\boldsymbol{\phi}}_{1}^{2}(\mathcal{K}, k)\right]-\widetilde{\boldsymbol{\phi}}_{1}^{2}(\mathcal{K}, k) P_{2}(\hat{\mathcal{K}} \cdot \hat{\boldsymbol{k}}),
$$

where $P_{2}$ is the Legendre polynomial.

The separation energy of the valence neutron(s) plays a crucial role in determining the relative importance of the Coulomb and nuclear breakup contributions [10]. In the breakup of ${ }^{11} \mathrm{Li}$ and ${ }^{11} \mathrm{Be}$, with separation energies of 0.3 and $0.5 \mathrm{MeV}$, respectively, the dominant reaction mechanism on heavy targets is Coulomb dissociation $[5,10,18]$. It is interesting, therefore, to consider the situation for ${ }^{6} \mathrm{He}$, with its larger two-neutron separation energy of order 1 $\mathrm{MeV}$. In doing so, however, it is critical to use realistic wave functions with the correct breakup threshold.

In the following we will use two ${ }^{6} \mathrm{He}$ three-body wave functions. These are calculated using the hyperspherical harmonic expansion method, and include hyperharmonics up to $K_{\max }=20$. The first (A) from Ref. [8], has a two-neutron separation energy of $0.975 \mathrm{MeV}$ and a root mean squared (rms) hyper-radius of $5.34 \mathrm{fm}$. Assuming an $\alpha$ particle rms matter radius of $1.49 \mathrm{fm}$, this yields a ${ }^{6} \mathrm{He}$ rms matter radius of $2.50 \mathrm{fm}$. The second wave function (B), calculated assuming a modified three-body interaction term, has separation energy $0.985 \mathrm{MeV}$, rms hyperradius of $4.935 \mathrm{fm}$, and produces a ${ }^{6} \mathrm{He}$ nucleus with a smaller rms matter radius of 2.35 $\mathrm{fm}$. These two wave functions, both with the correct breakup threshold, will allow us to gain a first impression of the sensitivity of the Coulomb breakup calculations to the assumed three-body wave function of ${ }^{6} \mathrm{He}$. The dominant angleindependent part of the projectile vertex $\mathcal{D}^{2}(\mathcal{K}, k)$ $=\left[\widetilde{\phi}_{0}^{2}(\mathcal{K}, k)+\widetilde{\phi}_{1}^{2}(\mathcal{K}, k)\right]$ calculated for wave function $(\mathrm{A})$ is presented in Fig. 2.

Recently, Balamuth et al. [19] have measured the $\alpha$-particle energy spectrum following the elastic breakup of ${ }^{6} \mathrm{He}$ on a $\mathrm{Au}$ target at a beam energy of around $65 \mathrm{MeV} /$ nucleon. The $\alpha$ particle was detected in a very restricted geometry at $5^{\circ}$ in the laboratory. The calculated laboratory differential cross sections $d^{2} \sigma / d E_{\alpha} d \Omega_{\alpha}$, as a function of $E_{\alpha}$ at $\theta_{\alpha}=5^{\circ}$, are shown in Fig. 3 (thin lines) together with the experimental data. The solid and dashed curves result from wave functions $\mathrm{A}$ and $\mathrm{B}$, respectively. In these calculations we integrate over all neutron solid angles in a forward cone of opening angle $20^{\circ}$. The calculated cross sections are seen to account for of order $50-70 \%$ of measured strength at the peak position. This agrees with the estimates of the Coulomb breakup contribution made in Ref. [19]. On the other hand the semiclassical Coulomb breakup calculations in Ref. [19], 


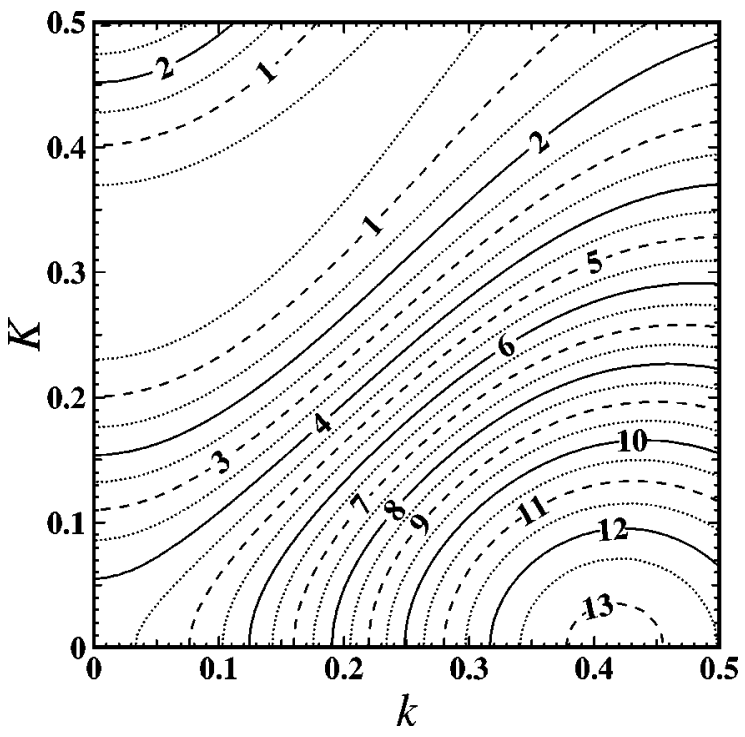

FIG. 2. Calculated vertex function $\mathcal{D}^{2}(\mathcal{K}, k)$ for wave function $\mathrm{A}$, in units of $10^{6} \mathrm{MeV}^{2} \mathrm{fm}^{6}$, as a function of $\mathcal{K}$ and $k$ in $\mathrm{fm}^{-1}$.

which assume a dipole transition and use the wave function of Danilin et al. [7], calculate a larger cross section.

We calculate the peak in the energy spectrum at around $264 \mathrm{MeV}$, slightly higher than the energy of the $\alpha$ particle corresponding to the beam velocity $(260 \mathrm{MeV})$, whereas the peak in the measured spectrum comes around $244 \mathrm{MeV}$. We understand that there are two reasons for this. First, the experiment was actually performed at $63.2 \mathrm{MeV} /$ nucleon. Secondly, the target thickness was such as to expect, typically, a $10 \mathrm{MeV}$ energy loss [20]. Upon simply shifting the calculated spectrum by $20 \mathrm{MeV}$ to compensate (thick lines), the peak positions are seen to be in satisfactory agreement. We conclude, as did the authors in Ref. [19], that the nuclear breakup contribution in these ${ }^{6} \mathrm{He}$ breakup data is substantial.

The grazing angle of incidence for the above reaction is around $3.5^{\circ}$. If it were possible to confine the measurements

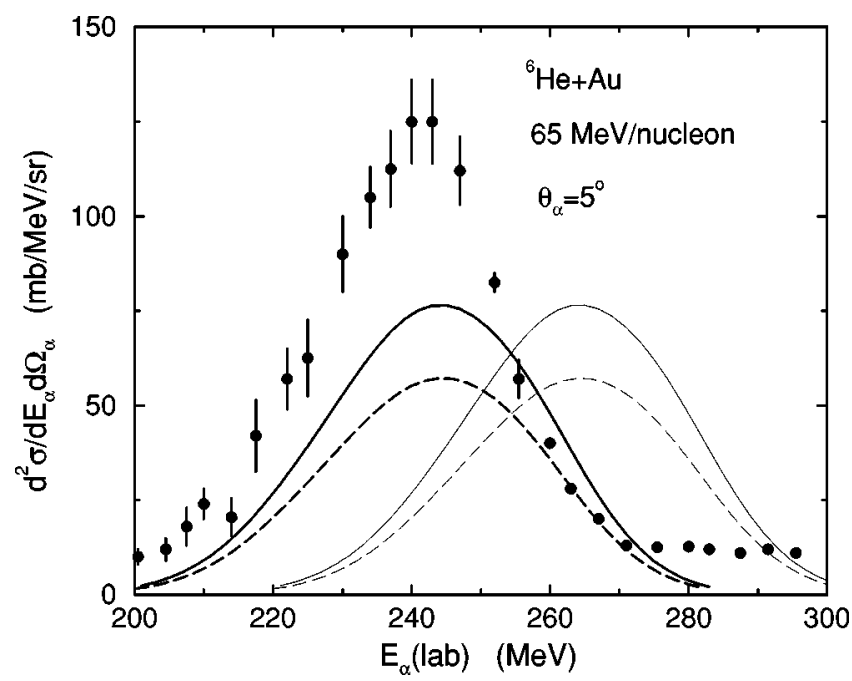

FIG. 3. Calculated $\alpha$-particle energy spectrum from Coulomb breakup of ${ }^{6} \mathrm{He}$ on $\mathrm{Au}$ at $65 \mathrm{MeV} /$ nucleon (thin lines). The solid and dashed lines use ${ }^{6} \mathrm{He}$ models $\mathrm{A}$ and $\mathrm{B}$, respectively. The experimental data are from Ref. [19]. The thick lines show the calculated energy spectrum shifted in energy by $20 \mathrm{MeV}$ (see text).

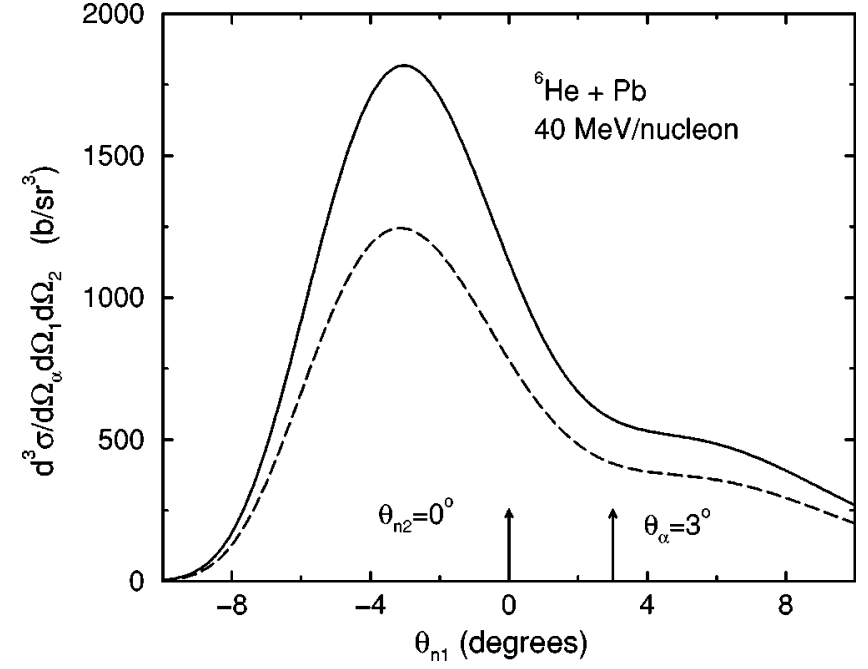

FIG. 4. Exclusive neutron angular distributions from the Coulomb breakup of ${ }^{6} \mathrm{He}$ on a $\mathrm{Pb}$ target at $40 \mathrm{MeV} /$ nucleon. The solid and dashed lines use ${ }^{6} \mathrm{He}$ models $\mathrm{A}$ and $\mathrm{B}$, respectively.

to more forward angles, below the grazing angle, it might be possible to measure physical observables which are less affected by nuclear breakup contributions. The exclusive neutron angular distribution measured at extreme forward angles, in coincidence with the $\alpha$-particle core and the second neutron, may be one such observable. In Fig. 4, we show predictions for such exclusive neutron angular distributions $d^{3} \sigma / d \Omega_{1} d \Omega_{2} d \Omega_{\alpha}$ as a function of $\theta_{1}$ from the Coulomb breakup of ${ }^{6} \mathrm{He}$ on a $\mathrm{Pb}$ target at $40 \mathrm{MeV} /$ nucleon. We use the two different wave functions for ${ }^{6} \mathrm{He}$ described above. The $\alpha$ particle is detected at a forward angle of $3^{\circ}$ and the other neutron at $0^{\circ}$. The grazing angle of incidence on this target at this lower beam energy is around $6^{\circ}$. There is a sideways asymmetry in the calculated neutron angular distributions, with more neutron cross section opposite to the alpha-particle detector. We see also that the magnitudes of the distributions are quite sensitive to the choice of wave functions, and therefore, such measurements can be related to the internal structure of the projectile.

The method developed here can also be used to calculate the dissociation cross sections as a function of the neutron- $\alpha$ relative energies, and also the angular correlations between the neutron and $\alpha$ particle fragments. The latter were the subject of recent experimental work at GSI [21]. This type of analysis is important in determining whether the breakup proceeds via resonances in the unbound $A-1$ systems, i.e., ${ }^{5} \mathrm{He}$ in the case of ${ }^{6} \mathrm{He}$ breakup. Calculations of these observables are also vital to establish the accuracy with which the resonant nature of the ${ }^{5} \mathrm{He}$ continuum is treated in the adiabatic approximation used here. Such calculations are in progress and will be presented elsewhere. We point out, however, that such calculations are beyond the scope of zerorange semiclassical and quantum-mechanical dineutron-core reaction models [9-11,22].

In summary, we have presented a theoretical model for approximate quantum-mechanical calculations of the elastic Coulomb breakup of two-neutron halo nuclei. The method permits a fully finite-range treatment of the three-body projectile breakup vertex and the use of realistic correlated three-body wave functions. We can thus take into account 
carefully the special structure features associated with halo nuclei. The approximations made are that (i) only the core particle is charged and interacts with the target, and that this interaction is a point Coulomb interaction and (ii) that the important excitations of the projectile are to the low-energy continuum, and so can be treated adiabatically. We have presented calculations for the Coulomb dissociation of ${ }^{6} \mathrm{He}$. These suggest that nuclear breakup contributions are sub- stantial in the kinematical region covered by the existing data.

The financial support of the United Kingdom Engineering and Physical Sciences Research Council (EPSRC) in the form of Grant No. GR/J95867 is gratefully acknowledged. The authors would like to thank Professor R. C. Johnson for stimulating discussions and suggestions.
[1] See, e.g., H. Toki, I. Tanihata, and H. Kamitsubo, Proceedings of the International Conference on Nucleus-Nucleus Collisions IV [Nucl. Phys. A533 (1992)].

[2] I. Tanihata, Nucl. Phys. A522, 275c (1991); T. Kobayashi, ibid. A553, 465c (1993), and references therein.

[3] P. G. Hansen and B. Jonson, Europhys. Lett. 4, 409 (1987); P. G. Hansen, Nucl. Phys. A553, 89c (1993).

[4] K. Ikeda, Nucl. Phys. A538, 355c (1992).

[5] D. Sackett et al., Phys. Rev. C 48, 118 (1993); K. Ieki et al., Phys. Rev. Lett. 70, 730 (1993).

[6] Y. Suzuki, Nucl. Phys. A528, 395 (1991).

[7] B. V. Danilin et al., Phys. Lett. B 302, 129 (1991).

[8] B. V. Danilin, I. J. Thompson, J. S. Vaagen, and M. V. Zhukov, Nucl. Phys. A632, 383 (1998).

[9] L. F. Canto, R. Donangelo, A. Romanelli, and H. Schulz, Phys. Lett. B 318, 415 (1993).

[10] R. Shyam, P. Banerjee, and G. Baur, Nucl. Phys. A540, 341 (1992); P. Banerjee and R. Shyam, ibid. A561, 112 (1993).

[11] C. A. Bertulani, G. Baur, and M. S. Hussein, Nucl. Phys. A526, 751 (1991).
[12] J. A. Tostevin et al., Phys. Lett. B 424, 219 (1998); J. A. Tostevin, S. Rugmai, and R. C. Johnson, Phys. Rev. C 57, 3225 (1998).

[13] R. C. Johnson, J. S. Al-Khalili, and J. A. Tostevin, Phys. Rev. Lett. 79, 2771 (1997).

[14] P. Banerjee, I. J. Thompson, and J. A. Tostevin, Phys. Rev. C 58, 1042 (1998), this issue.

[15] G. Baur and D. Trautmann, Nucl. Phys. A191, 321 (1972).

[16] A. Nordsieck, Phys. Rev. 93, 785 (1954).

[17] J. S. Al-Khalili, I. J. Thompson, and J. A. Tostevin, Nucl. Phys. A581, 331 (1995).

[18] T. Nakamura et al., Phys. Lett. B 331, 296 (1994).

[19] D. P. Balamuth, K. A. Griffioen, J. E. Bush, K. R. Pohl, D. O. Handzy, A. Aguirre, B. M. Sherrill, J. S. Winfield, D. J. Morrissey, and M. Thoennessen, Phys. Rev. Lett. 72, 2355 (1994).

[20] J. E. Bush (private communications).

[21] L. V. Chulkov et al., Phys. Rev. Lett. 79, 201 (1997)

[22] S. Typel and G. Baur, Nucl. Phys. A573, 486 (1994). 